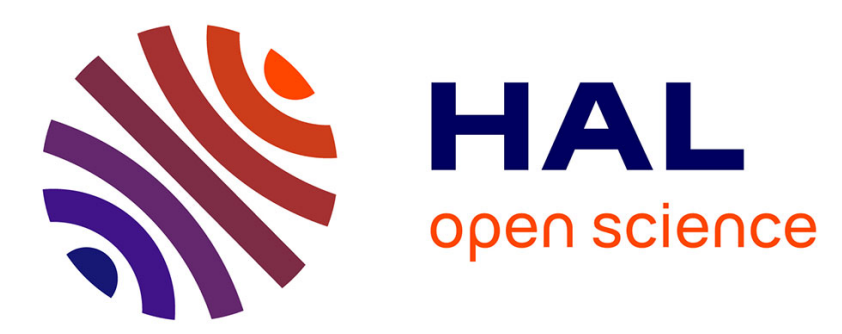

\title{
Studies on the written characters orientation and its influence on digit reversal by children
}

\author{
Jean-Paul Fischer
}

\section{To cite this version:}

Jean-Paul Fischer. Studies on the written characters orientation and its influence on digit reversal by children. Educational Psychology, 2017, 38 (5), pp.556-571. 10.1080/01443410.2017.1359239 . hal-02024612

\section{HAL Id: hal-02024612 \\ https://hal.univ-lorraine.fr/hal-02024612}

Submitted on 19 Feb 2019

HAL is a multi-disciplinary open access archive for the deposit and dissemination of scientific research documents, whether they are published or not. The documents may come from teaching and research institutions in France or abroad, or from public or private research centers.
L'archive ouverte pluridisciplinaire HAL, est destinée au dépôt et à la diffusion de documents scientifiques de niveau recherche, publiés ou non, émanant des établissements d'enseignement et de recherche français ou étrangers, des laboratoires publics ou privés. 


\title{
Studies on the written characters orientation and its influence on digit reversal by children
}

\author{
Jean-Paul Fischer \\ InterPsy Laboratory, University Lorraine, Nancy, France
}

\section{ABSTRACT}

Recent research has found that children reverse mainly the leftoriented characters when writing from memory (e.g. they write $\mathrm{I}$ and $\varepsilon$ instead of $J$ and 3 ). In order to obtain an objective definition of the left-orientation of a character, the ratings of the level of leftorientation of all the asymmetrical capital letters and digits by 142 adult students was analysed in Study 1. Study 2, on 298 five-sixyear-old children, examined an immediate prediction of Study 1 , namely that the children reverse mainly the digits that the adult students have rated left-oriented. Other predictions, both of the posited representation of the writing during the reversal stage and the neurological process of mirror generalisation, were verified: the simplicity of the representation of the symmetrical digits 0 and 8 makes incorrect writings very rare; the mirror generalisation, which operates only in the left-right direction, makes other transformations (inversion or $180^{\circ}$ rotation) very rare. Finally, the explanatory power of some putative individual factors of reversal (e.g. writing with the left hand) is shown to be far lower than that of the left-orientation of the characters.

\section{ARTICLE HISTORY}

Received 3 March 2017 Accepted 20 July 2017

\section{KEYWORDS}

Mirror writing; mirror generalisation; reversal; inversion; rotation; handedness

\section{A short historical background}

Reversal of characters - letters and digits (for digits see Figure 1) - in the writing of the typical developing child is a phenomenon know at least since the beginning of the twentieth century. This is testified by Fuller (1916) who, speaking on mirror writing, of which left-right reversal of the characters is a component, wrote that 'nearly every child at a certain period of its development will be found to produce spontaneous, fragmentary mirror-writing with the right hand' (p. 201). However, the fact that neuropsychologists and developmental psychologists have thought having a 'good' explanation of the phenomenon - the left-handedness of the children - has restricted the spreading of this information (incompatible with the good explanation) and prevented the emergence of another explanation. On the contrary, the paper by Fischer and Koch (2016a), in questioning and even rejecting the explanation by hand of writing, encourages the exploration of another explanation. Such other explanation was already suggested by Simner (1984) who remarked that children'reverse the left-facing letters more often than the right-facing letters' (p. 141). 


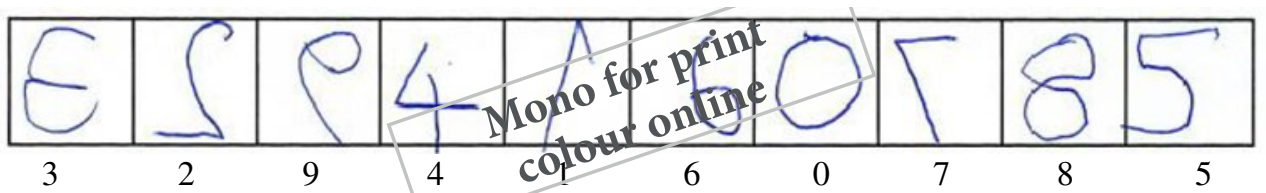

Figure 1. An example of a series of the 10 digits written with the (preferred) right hand by a 5 years 10 months-old girl (the writings of 3, 2, 9, 1 and 7 are reversed).

This explanation was recently confirmed and expanded by Fischer and coworkers (Fischer, 2013, 2017; Fischer \& Koch, 2014, 2016b; Fischer \& Tazouti, 2012), Treiman and coworkers (Treiman \& Kessler, 2011; Treiman, Gordon, Boada, Peterson, \& Pennington, 2014), and by Mclntosh and Della Sala (2012) in a more general paper on 'mirror writing'.

\section{A recent theoretical account}

Fischer and coworkers explained reversal of the characters via a right-writing rule, which could be acquired by statistical learning (Treiman \& Kessler, 2011; Treiman et al., 2014). Due to mirror generalisation by the brain (Corballis \& Beale, 1976; Dehaene, 2010), four-five-yearold typically developing children know only the shape of the characters but not their horizontal (left-right) orientation. Consequently, in our left-to-right-oriented culture, the right-writing rule predicts that children should prefer to orient their writing rightwards when writing the characters from memory; therefore, they reverse mainly the left-oriented characters $(1,2,3,7,9, \mathrm{~J}$, and Z; see also Figure 1). However, this theoretical account poses the problem of an objective definition of the left-orientation of a character and does not describe the way children represent the shape of the characters without orientation.

\section{How do children represent the writing of a character without its orientation?}

Given the visual nature of the process of mirror generalisation, the theoretical account suggests that children could represent the shape in a visual image. However, the latter suggestion runs in difficulty for at least two reasons. First, a visual image without orientation is difficult to conceive, unless one follows the old proposition by Orton (1925) that there is a correct memorised visual image of each character in one cerebral hemisphere, and a symmetricalimage of the same character in the other hemisphere. Second, even as young as 4 or 5 years, children recognise (and often produce) the writing of the characters irrespective of their size, visual aspect (e.g. font, colour), location (e.g. removable characters) and viewing angle. The explanation of the latter capacity rejoins the problem of pattern recognition by humans (Shapley, Caelli, Grossberg, Morgan, \& Rentschler, 1990). Thus, the representation of the writing of the character cannot be restricted to the knowledge of the shape of a character in a specific font; it should be more abstract.

Our theory posits that this abstract representation of the writing of a character results from the identification of a set of invariants features of that character (cf. Burgoon, Henderson, \& Markman, 2013). Schubert (2017) proposes a systematic analysis of these features for the capital letters and digits. Referencing to this analysis, the second column of Figure 2 describes the hypothesised features of the representation of the digits during the stage of reversal, when children's knowledge is restricted to the non-oriented shapes. Because the process of 


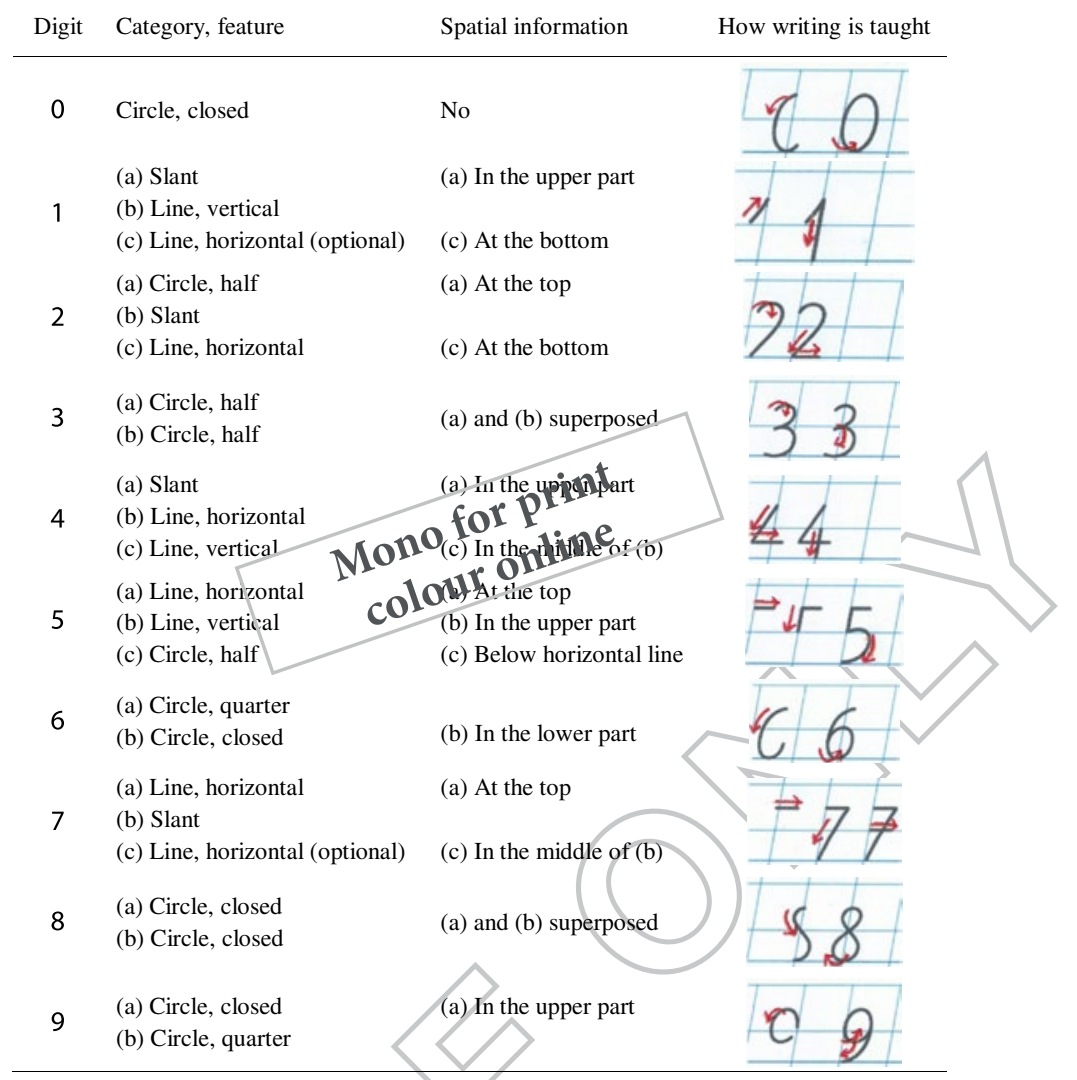

Figure 2. The hypothesised abstract representation of the 10 digits during the reversal stage, and the teaching of their writing in French school [cf. MEN (2013): the digits are slightly biased towards right because the French school system emphasises on cursive writing].

mirror generalisation deletes only the left-right orientation, the abstract representation of the characters can nevertheless include some other spatial information, notably the vertical position. In the third column of Figure 2, such other spatial information was added to the features in the second column. This spatial information allows children to avoid many erroneous writings. For example, without the spatial information, they could produce the digit 8 by drawing two horizontally adjacent circles: This would result in a digit 8 that is lain down on its side $(\infty)$.

These abstract representations allow distinguish between very nearly identical characters. For example, the distinctive features are the horizontal stroke in the pair $\{C, G\}$, the slant in the pair $\{P, R\}$, or the bottom horizontal stroke in the pair $\{F, E\}$ (cf. Reed, 2016). However, the abstract representation does not allow to identify the correct 3 in the pair $\{\varepsilon, 3\}$ or the correct $\mathrm{R}$ in the pair $\{Я, R\}$.

\section{How do children orient characters?}

Certain perceptual features suggest a left- or right-orientation for some characters. For example, the left openness of the two half circles of 3 make this digit facing left; the right openness of the quarter circle of 6 makes this digit facing right. However, for many other characters, 
the perceptual features are not sufficient to indicate a left- or right-orientation. Notably the left- or right-orientation of a horizontal stroke, or of a slant, depends on the starting point. Therefore, the dynamic of writing, as it is taught in the French école maternelle, was added in the last column of Figure 2. The combination of the dynamic of writing, as suggested by teaching, with the abstract representation sometimes shed light on the orientation of a digit. For example, when drawing a 4, French (or western) people always draw the acute angle (open towards the right) first, and then lift the pen before drawing the vertical on the right of the vertex of the angle. Given this writing dynamic, 4 may be categorised as right-oriented, even though the static form of the finished digit cannot be said to have any specific orientation.

However, people may not always write characters in the way they were initially taught. For example, French people do not systematically respect the left-to-right writing of the horizontal stroke in 5 because they often prefer begin the writing at the top-right in the aim avoiding lift the pencil. This, added to the subjectivity and incompleteness of the preceding analyses, left the problem of the character orientations unsatisfactorily solved. To solve it better, the solution is probably that used by Treiman et al. (2014), that is, to request rating of the orientation by adult subjects. This was the solution retained in Study 1 , with French participants because they have probably been submitted to the same initial learning as the children of Study 2.

\section{Some predictions}

The theory posits an abstract representation, without orientation, of the writing of the characters, before memorisation of the orientation by the five-year-old children some months later. The features of the digits described in Figure 2 show that the representations of 0 and 8 are probably the simplest ones; particularly, that of 0 when one knows that the drawing of circles, or'rounds' (ronds in French) as say the children, is trained precociously. Both because of this simplicity, and because 0 and 8 are symmetrical digits that do not need a memorisation of the left-right orientation, a first prediction should be that the digits 0 and 8 generate a higher proportion of correct writings than the other digits. Note that here, and also throughout the paper (if no other indication) the qualifier symmetrical (or similar) means symmetric about a vertical axis.

A second prediction of the theory should be that the children make mainly left-right orientation errors. The process of mirror generalisation operates only for the left-right orientation. A classical argument in favour of this specificity of the mirror generalisation process was found in the human evolution. 'Animals may appear in mirror-opposite profiles, danger may lurk on either side', remarked Corballis and Beale (1976, p. 25), whereas an animal 'is less of a threat upside down than right side up' (Rollenhagen \& Olson, 2000, p. 1506) or when the animal turns around to go away. Likewise, for the infant, seeing the left or right profile of the mother is unimportant, whereas distinguish the head from the foot, and if the mother comes or goes away, seem fundamental. Thus, through the specificity of the mirror generalisation process, children should produce a great number of reversed writings (symmetrical relative to a vertical axis in the plane), but far less inverted (symmetrical relative to a horizontal axis in the plane) or $180^{\circ}$ rotated (also resulting from performing consecutively the two preceding symmetries) writings. 
A third prediction should be that the left-right character orientation, as rated by adult persons, correlates positively with the left-right reversals produced by the children. To our knowledge this was never verified for digits and is a main motivation of the present research.

Finally, the theory claims that the main explanation of reversals in children is the orientation of the characters. Therefore, the explanatory power by individual factors must be lower than the explanatory power by this feature of the items. This should principally be the case of the explanation through left-handedness, which was the major explanation in the twentieth century as mentioned in our brief historical introduction, whereas the explanation through gender (Hildreth, 1950, p. 69) is only secondarily targeted. Thus, a fourth prediction should be that the part of variance in the data explained by the participants' hand of writing is much lower than the part of variance explained by the character orientations. The same holds for gender if this predictor explains a significant part of variance.

\section{Organisation of the research}

In order to test these predictions two studies were designed. In the first study, the adult's rating of the left-right orientation of the characters was established. This study precedes the second study in which children wrote the digits in a dictation task. In spite of the difference between participants - adults vs. five-year-old children - the two studies are linked by the fact that the examination of our third and, to a lesser degree, our fourth predictions in the second study use the result of the first study. However, there is a minor difference between the two studies with respect to the material: The first study examined characters capital letters and digits - whereas, the second study was restricted to the digits. One reason of this comes from the fact that 11 out of the 26 capital letters are symmetrical and therefore, cannot be differentiated from their reversal; another reason comes from previous results that showed that children reverse (frequently) only two letters, J and Z (Fischer, 2011). Thus, in a study of the 36 characters ( 26 letters +10 digits), a floor effect could affect the percentages of reversal, with an accompanying right-skewed distribution of this percentage. Furthermore, restricting the study to the 10 digits gives the possibility to obtain many writings of a same digit, both with good ecological validity and in a unique experimental session. Given the instability of the reversal phenomenon, four writings of each digit confer a more stable measure of the frequency a child reverses a digit.

\section{Study 1: rating orientation of the characters by adult students}

\section{Method}

\section{Participants}

The participants were 142 undergraduate students, Mage $=21.8$ years, SD $=4.2$, including, 27 males and 18 students who wrote with the left hand. They participated voluntarily as part of a psychology tutorial group.

\section{Material}

Each participant had an A4 sheet (recto/verso) with the 24 characters - 15 asymmetrical capital letters, eight asymmetrical digits, and one symmetric character (the digit 8) included as control - displayed in six lines of two characters on each side of the sheet. The characters 
were written in bold, size 14, with the 'Script cole' font (except Q, which was in Arial Unicode MS). Fifteen different random orders of the 24 characters were proposed, approximately in the same proportion to the students. Each character was associated with five checking-boxes, with the indications from left to right, 'clearly left', 'rather left','neither left nor right', 'rather right', and 'clearly right', respectively.

\section{Procedure}

After a succinct explanation of the aim of the multiple choice questionnaire, two contrasting examples with lower-case letters were provided, but were not lengthy discussed. Students in a group - there were seven groups of unequal size - were informed that there is no time limit, but that their first impression is generally the best. The experimenter emphasised they should carefully indicate their choice for each of the 24 characters in the order of usual writing direction, that is, from left to right and from top to bottom. This emphasis seems to have being effective as the number of omissions was low (less than .3\%).

\section{Coding}

15 Five, four, three, two and one points were attributed to the five checking-boxes associated with a character, respectively. Thus, the mean of the points attributed to a given character measures its degree of left-orientation, a high mean (up to five) corresponding to a high level of left-orientation of this character.

\section{Results}

20 The mean of the points for the 24 characters are represented in Figure 3. Beforehand, it is interesting to note that the mean 2.951 of the symmetrical character ' 8 ' is very near from three points, the median of the coding points: The absolute value of its difference with three is .049. In contrast, all the 23 differences between the mean of the asymmetrical characters and three are greater (in absolute value) than .169. With a false discovery rate method of control for multiple comparisons, all ps, except the $p$ associated with 8, are lower than .005 .

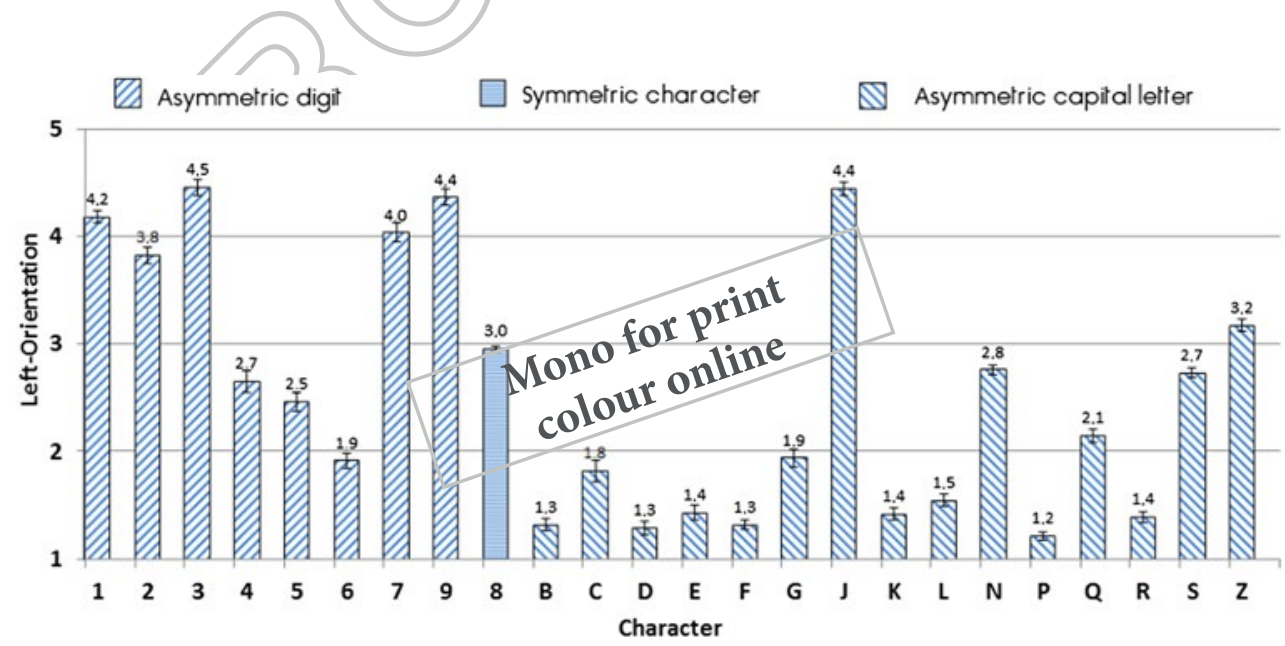

Figure 3. Rating of the character orientation by 142 adult students on a five levels Likert scale: $5=$ extremely left-oriented, 1 = extremely right-oriented (error bars represent \pm 1 SEM). 
In the perspective of Study 2, it is important to see, on Figure 3, that among the asymmetrical digits, the subset $L O=\{1,2,3,7,9\}$ and its complementary $R O=\{4,5,6\}$ are characterised by a rating of left-orientation above and below the median 3 , respectively. The 'distance' between the two subsets is significant because the lowest rating of a LO digit (that of 2: 3.824) is greater than the highest rating of a RO digit (that of $4: 2.652$ ): $t(140)=8.304$, $p<.001, \mathrm{Cl}_{95 \%}=[.886,1.440]$. Furthermore, the digit 4 is not only rated right-oriented (slightly but significantly), but it is also nearer from 5 , which is the nearest right-oriented digit, than from 2, which is the nearest left-oriented digit.

\section{Discussion}

10 A comparison with the ratings reported by Treiman et al. (2014) is possible for the uppercase letters. Exactly as in the current study, the 33 students (at Washington University in St Louis) of Treiman et al. rated as facing left only the two uppercase letters J and Z. More generally, the left-facing or right-facing ratings correlate highly between the two studies, $r_{\mathrm{p}}(13)=.925$, $p<.001$. Given the differences in participants, procedure, and writing of the uppercase letters in the two involved cultures, such a high correlation is noteworthy.

For the digits, the rating of the adult students clearly differentiates $\mathrm{LO}$ and RO digits. However, despite the significant result showing that adult students rate the digit 4 as right facing, we would not claim that the closed (at the top) writing of the digit 4 faces right, or points towards the right. In fact, the question of the orientation of the digit 4 is complicated by its teaching: Teachers teach an open 4 (see Figures 1 and 2). Moreover, in the common writing, the acute angle of 4 often closely resembles a right angle.

\section{Study 2: children's reversal of the digits}

\section{Method}

\section{Participants}

25 Participants were children recruited from 18 upper section classes of the French école maternelle. All standard administrative authorisations and ethical rules for such a study were respected. All children present at the moment of the test, and who were judged able to participate by their teacher, participated. However, 26 children, out of the 324 in the initial sample, produced correct writings for all the digits (40 out of 40 ). Most likely these children were developmentally beyond the stage of reversal, and were excluded from the present analyses. Finally, the analysed sample was composed of 298 children: 146 girls, 152 boys, $M_{\text {age }}=5.74$ years, $\mathrm{SD}=.32$, age range: $5.09-6.48$. Twenty-nine children spontaneously wrote with their left hand and 269 with their right hand.

\section{Material}

The children had to write four series of the 10 digits. In order to write a series, they had a sheet of paper (half A4 format) with 10 aligned adjacent boxes (see Figure 1). Each box measured $2 \times 2 \mathrm{~cm}$. The order of digit was generally not the natural order and was not the same for all children. In fact, there were four sets, each composed with four series, all 16 involved series differing with respect to the order of the digits. Furthermore, when a same set of series was given to two groups of children, the order of the four series was generally 
not the same. Thus, there was a great variation in the order of writing the digits, although this variation was not systematic.

\section{Procedure}

Three experimenters tested the children in groups of four $( \pm 2)$, near their classroom but apart from other children. Children were seated as far apart as possible to prevent copying. They were told they had to simply write the dictated digits in the successive boxes. If necessary the experimenter indicated the first box (furthest left) by pointing towards a child's sheet of paper.

The experimenter stressed that digits must be written in the boxes in the order of dictation, that no boxes should be omitted, and that copying was forbidden and unnecessary. If children did not know how to write a digit, they could simply make a cross in the box. Between the dictations of two series, the experimenter collected the preceding sheet in order to avoid a copy or any other influence of a previous writing.

\section{Coding}

15 First, the experimenters judged the children's writings and coded them into five categories, and then another judge independently coded the writings into the same five categories. The categories were: (1) correct (readable); (2) left-right reversal (see, for example, the writings of 3, 2, 9, 1 and 7 in Figure 1); (3) upside-down inversion; (4) $180^{\circ}$ rotation (a $180^{\circ}$ rotation of 9 looks like a 6, and conversely); and (5) other (omission, cross, other digit, unreadable and intermediate writing). Correct production of the shape of the digits was defined very liberally; however, in order to be classified into one of the four first categories, the transformation of the writing (reversal, inversion or rotation), or not (correct writing), had to be unequivocally recognisable. Concordance between the first and second judgments was $98.8 \%$. The $1.2 \%$ conflicts were resolved by discussion between the second judge and an experienced primary school teacher.

\section{Data processing}

The percentage of correct writing of a character, or by a child, was computed relative the total opportunities of writing this character. But the percentage of transformed writings (reversed, inverted or rotated) was computed as the number of transformed writings divided by the number of relevant writings (all the correct and transformed ones). We did this because it does not make sense to study the transformation of digits the children could not write at all.

The verification of Prediction 4, on the relative importance of different factors favouring or explaining digit reversal, involves a modelling with the R-programme (R Core Team, 2015). Because three observed factors - the biological factors gender, age and hand of writing concern the participants, and one factor - orientation of the digits - concerns the items, a linear mixed modelling seems relevant. Indeed, an important advantage of a mixed model is the possibility to include simultaneously predictors that are tied to the items and predictors that are tied to participants (Baayen, Davidson, \& Bates, 2008).

To construct a first type of mixed models, the percentage reversal was computed for each participant and each number. When a child has not produced any correct or transformed writing for a digit, this percentage was not available (NA) for this digit. There were 15 such NA codes in the $298 \times 8=2384$ cells of this first datasheet (i.e. less than $.63 \%$ ). The Imer 
function of the Ime4 R-package (Bates, Maechler, Bolker, \& Walker, 2015), which easily handles missing data, was preferred for the fit of the model (by REML: restricted maximum likelihood).

To construct the second type of mixed models, the percentage reversal was computed for each participant and each class of digit (cod) - LO and RO - determined in Study 1. There was no NA code in the $298 \times 2=596$ cells of this second datasheet. Therefore, the Ime function of the nmle R-package (Pinheiro, Bates, DebRoy, Sarkar, \& R Core Team, 2017), which fits the model only by REML, was used.

\section{Results}

10 Predictions $1-3$

Prediction 1, that is, a greater percentage of correct writing for the digits 0 and 8 than for any other digit, was clearly confirmed. The digits 0 and 8 generated 99.58 and $94.97 \%$ correct writings, respectively. This is more than the greatest percentage $-84.40 \%$ for the digit 4 - of correct writing of any other digit. In addition, these percentages are far greater than the most correct written left-oriented digit, $74.41 \%$ for the digit 1 . Such a restriction of the comparison to the left-oriented digits is justified if one assumes that children who applied the right-writing rule in fact wrote the non-left oriented digits $(4,5$ and 6$)$ correctly by chance.

In order to verify that children produce more reversed than inversed or rotated transformations (Prediction 2), the symmetrical digits 0 and 8 , and the digit 3 (symmetric around a horizontal axis), were excluded from the analysis because correct, inverted, rotated, and reversed writings of these digits cannot always be distinguished. Prediction 2 was also clearly confirmed. The percentages of inverted, rotated, and reversed digits were $.89,1.26$ and $24.88 \%$, respectively. Thus, the percentage of reversal is from another order of magnitude than that of inversion or rotation.

Finally, Prediction 3, that of a positive correlation between the left-orientation ratings by the adult students (in Study 1) and the reversals by the children, also was clearly confirmed. Children reversed 39.0,34.6, 30.5, 30.0, 24.7, 21.7, 20.7 and $11.9 \%$ of the writings of 3, 9, 7, $2,1,6,5$ and 4, respectively. Therefore, (see Figure 3), the positive correlation was high and significant, $r_{\mathrm{p}}(6)=.788, p=.010, \mathrm{Cl}_{95 \%}=[.32,1.00]$ with a one-tailed test. The large confidence interval is due to the small degrees of freedom and therefore is not worrisome. Furthermore, to eliminate the explanation of this strong correlation by a peculiarity of the distributions of the two correlated variables, Spearman's rho was also computed: $r_{\mathrm{s}}(6)=.833, p=.008$ (one-tailed).

\section{Prediction 4: a preliminary linear modelling analysis}

35 In order to evaluate our hypothesis that the items explain better the reversal data than the participants with a linear mixed model it is crucial to construct an adequate random effect structure for this model. Therefore, a simple classical multiple regression analysis was preliminary performed with all available predictors (hand, gender and age) and with the individual percentage of reversal as the dependent variable. The resulting model was significant, $F(3,294)=2.94, p=.033$. But the model explains only $2.9 \%$ ( $1.9 \%$ with an adjusted $R$-squared) of the variance. Thus, a large place remains for other explanations.

As regard to the three predictors, hand has a significant effect $(p=.019)$; children who write with the left hand were estimated produce $8.9 \%$ more reversals than children who 
write with the right hand. Gender has a marginally significant effect ( $p=.073$ ); girls were estimated produce $4.0 \%$ more reversals than boys. Age has no effect ( $p=.891)$. Given the effects of hand and, secondarily, gender, the data were further analysed to determine if these two differences between subgroups are only quantitative or also qualitative. Namely is the hierarchy of reversal frequency of the eight asymmetrical digits the same in the left-hand subgroup as in the right-hand subgroup, by the girls as by the boys?

The children who wrote with their left hand showed the same pattern of reversal frequency of the different digits that the children who wrote with their right hand: The computation of the correlation between reversal of the eight digits in the two sub-samples yields $r_{\mathrm{p}}(6)=.924, p=.001$; in both subgroups the digit 3 is the most reversed and the digit 4 is the least reversed. The same holds for the comparison between girls and boys: $r_{p}(6)=.960$, $p<.001$. Thus, it seems unnecessary to complicate a mixed model with a random-slope component.

\section{Prediction 4: a first mixed model analysis}

15 A mixed model was computed with the Imer function applied to the first datasheet (see data processing, in the method section). First, a null model (M0.8Dig), with no fixed effect but only random-intercept for subjects (298 participants) and items (eight digits) was computed. Then the hand predictor was added as fixed effect (M1.8Dig), and, subsequently, the gender predictor also (M2.8Dig).

The results for the fixed effects confirm that of the preceding simple linear analysis and are like that of the following mixed analysis (thus, they are not reported here). The results for the random effects are reported in Table 1. First, not surprisingly, Table 1 shows that the by-item random-intercept variance does not change, whether or not the fixed effects of hand and gender are included in the model. Second, the reduction of variance by introducing the hand predictor can be estimated at 227.6 - 222.8 $=4.8$ by comparison of the M0.8Dig and M1.8Dig models. This reduction, when compared with the constant (between models) 67.5 by-item random-intercept variance, is considerably less, and not of the same order of magnitude (about 14 times less). Likewise, in comparing M2.8Dig to M1.8Dig, the impact of gender on random-intercept variance can be estimated about 20 times lesser than the impact

of the eight digits.

\section{Prediction 4: a second mixed model analysis}

The first mixed model analysis does not take into account the important distinction between LO and RO digits established in Study 1 . Another mixed model was therefore, computed on the second datasheet (see data processing, in the method section). Like before, three nested

Table 1. Variance of the by-participant and by-item random-intercept in three nested mixed models computed with eight digits as items.

\begin{tabular}{lrrr}
\hline Group & \multicolumn{3}{c}{ Random-intercept variance } \\
\hline Model & M0.8Dig & M1.8Dig & M2.8Dig \\
\hline Participants & 227.6 & 222.8 & 219.8 \\
Items (eight digits) & 67.5 & 67.5 & 67.5 \\
Residual & 1139.4 & 1139.3 & 1139.3 \\
Total & 1434.5 & 1429.6 & 1426.6 \\
\hline
\end{tabular}


Table 2. Variance of the by-item random-intercept in three nested mixed models computed with two classes - left-oriented and right-oriented - of digits as items.

\begin{tabular}{lccc}
\hline Group & \multicolumn{3}{c}{ Random-intercept variance } \\
\hline Model & M0.2Cod & M1.2Cod & M2.2Cod \\
\hline Orientation & 92.2 & 92.2 & 92.2 \\
Residual & 716.1 & 711.7 & 709.1 \\
Total & 808.3 & 803.9 & 801.3 \\
\hline
\end{tabular}

models M0.2Cod (no fixed effect), M1.2Cod (hand as fixed effect), and M2.2Cod (hand and gender as fixed effects) were computed.

The model M2.2Cod leads to the two following estimates: (1) a child who writes with the left hand produces $8.0 \%$ more reversals than a child who writes with the right hand, $t(592)=2.18, p=.03, \mathrm{Cl}_{95 \%}=[.80,15.26] ;(2)$ a girl produces $3.9 \%$ more reversals than a boy, $t(592)=1.79, p=.07, \mathrm{Cl}_{95 \%}=[-.38,8.19]$ and $\mathrm{Cl}_{90 \%}=[.31,7.50]$.

Table 2, which represents the random-intercept variance, needs a prior explanation. Because each participant has only two measures - the orientation percentages $L O$ and RO there is no by-participant random-intercept variance. Indeed, the by-participant variance is completely confined in - and confused with - the by-item variance. Table 2 shows that the introduction of the fixed effect of hand in the M1.2Cod model reduces the residual (or total) variance by $716.1-711.7=4.4$. This reduction, due to the hand predictor, is very low compared to the constant (between models) by-item random-intercept variance, and is not of the same order of magnitude (about 20 times less). Likewise, in comparing M2.2Cod and M1.2Cod, the impact of gender on random-intercept variance can be estimated about 35 times lesser than the impact of orientation ( $\mathrm{OO}$ vs. RO).

\section{Discussion}

A specific prediction of the whole research was that the left-orientation ratings of the adult participants in Study 1 correlate with the children's reversals in Study 2. This was clearly the case for the eight asymmetrical digits. Even if the correlation is not perfect it respects the separation between LO and RO digits: The five LO digits are the five most reversed digits and the three $\mathrm{RO}$ digits are the three least reversed digits.

The importance of the digit orientation in the explanation of their reversal by the children was also quantitatively supported by comparison of mixed models in which a randomintercept variation of the digits was introduced. This comparison shows that the fixed effect of the hand of writing on reversal is far lower and not of the same order of magnitude as the random-intercept effect of the digits. Furthermore, these comparisons show that when the digits are regrouped in classes of digits - left-oriented and right-oriented - the proportion random-intercept variance due to the two classes of items is greater than when the digits are not regrouped.

The very high percentage of correct writing of 0 (near of 100\%) and 8 (about 95\%) does not mean that these writings were, a priori, trivial. Indeed, the difficulty of the mathematical concept of zero could make this number less known by the children. This because conceptual knowledge correlate with procedural (writing) knowledge, even if these two kinds of knowledge are distinct as demonstrated in the domain of emergent literacy by Puranik and Lonigan (2014). For the digit 8, the performing of the writing without lift of the pencil is a very difficult 
motor learning. Our results should not suggest that performing the writing of 8 is easily overcome by five-year-old children. In fact, many children produced distorted writings (coded as correct provided that the writing is unambiguously recognisable), whereas other produced the writing 8 by superposition of two circles (also coded correct).

Another prediction of the theory, of the process of mirror generalisation precisely, is that transformations (inversion and rotation) other than left-right reversal should be very rare. This result was clearly confirmed, as other transformations are far less numerous (about 10 times less even when rotations and inversions are added) than left-right reversals. The finding can appear as a known result. However, the knowledge results rather from observational data than from experimental studies. The data reported by Lewis and Lewis (1965) relate to first-graders submitted to a copying task. Despite these major differences, in participants and procedure, with the current study the data show that reversals were, approximately 5 and 30 times more numerous than inversions at the first school-year pre-instruction and post-instruction test, respectively (ratios computed from the Table 4 in Lewis and Lewis). Fischer and Koch (2014) also report only inversion data. Although not directly comparable, because the material included both the capital letters and digits, Fischer and Koch report a very low percentage of inverted character compared to that of reversed characters, $0.2 \mathrm{vs}$. 18.8. Thus, the current finding of a very low frequency of inversion of digit writings by children corroborates the previous findings. However, the estimation of the frequency of rotations is rare in the literature. An analogous absence was pointed by Erlikhman, Strother, Barzakov, and Caplovitz (2017). They noted that, since the work by Kolers and coworkers in the 60 s or 70s (e.g. Kolers \& Perkins, 1975), no study was aimed to understand the relative difficulty of reading different transformations of a text. Notably, the question why rotated text (i.e. reflected twice) is easier to read than mirrored text (i.e. only reflected once).

\section{General discussion and conclusions}

The present research clearly supports the general hypothesis that the perceived orientation of the characters (capital letters and digits), in a culture in which writing runs from left to right, explains in great part the reversal of the left-oriented digits by the five-year-old children. This result paves the way for further analysis of children's representations of characters, including neuropsychological aspects of these representations, when they begin learning to read and write. It also opens up discussion of the origin of the perceived orientation of characters in western cultures and provides a stepping stone for estimating the relative influence of other factors that may explain character reversals by young children.

The neuropsychological finding that the brain mirror generalises the visual images (Dehaene, 2010; Fischer \& Koch, 2014; Rollenhagen \& Olson, 2000) allows a greater understanding of the phenomenon reversal of the characters by children. Because mirror generalisation performs only a left-right symmetry (around a vertical axis in the plane), two simple predictions of this restriction were verified: (1) the (left-right) symmetrical digits 0 and 8 were more correctly written than any other digit; (2) children made very few inversion (symmetry around a horizontal axis) and $180^{\circ}$ rotation (two successive symmetries around orthogonal axes) errors.

Mirror generalisation results in a representation of the characters without their left-right orientation in five-year-old children. What such a representation could be is an unsolved question. A theoretical argument suggests that the representation should be somewhat 
abstract. In Figure 2, a description of the representation without orientation of the digits is proposed. Because this representation is static, the dynamic of writing, as it is taught in the French école maternelle, was added. This assumed abstract representation is difficult to reconcile with Orton's (1925) suggestion of a two-fold representation of the character, one with the correct orientation, typically in the left hemisphere, and another with the reversed representation, typically in the right hemisphere. The idea of such a two-fold representation is always present in the neuropsychological literature, taking often the proposal of a 'dormant' representation of the reversed writing in the adult's right hemisphere (Kushnir, Arzouan, Karni, \& Manor, 2013). However, the recent research by Borst, Ahr, Roell, and Houdé (2015) on expert readers does not support the two-fold representation (and its role in reversal). Indeed, the results of the research by Borst et al. argue against the assumptions of models in which 'mirror errors occur because of the automatic co-activation of the representation of a letter in the language hemisphere and the representation of its mirrorimage in the language non-dominant hemisphere's homotopic area' (p. 233).

When combined with the dynamic of the taught writing, the abstract representation can explain some reversals. For example, because children learn to draw the circle counterclockwise, the representation predicts reversal of the digits 2, 3 and 9, and no reversal of the digits 6 and 5 (the digit 5 because the half circle must be below the horizontal stroke), if children begin the drawing at the top. But the latter condition is not always satisfied. For example, direct observation shows that children often begin the drawing of the digit 1 at the bottom (Fischer, 2010). This can explain why the digit 1 is often reversed: children who begin the drawing at the bottom and then apply the right-writing rule, will reverse the digit 1 . The same holds for digit 7. In order to make quantitative predictions on the frequency of reversal of the different digits, the percentages of children who begin the drawing, as suggested by teaching, and who then apply the right-writing rule, should be stable and precisely known. This is presently not the case. Therefore, many authors (e.g. Fischer \& Koch, 2016b; Treiman et al., 2014) prefer the notion of left-facing and right-facing (or left-oriented and rightoriented) already used by Simner (1984) to categorise the characters. Because these notions are not well defined, the method of rating by adult participants seems to be the most appro30 priate to obtain an objective categorisation.

In the first study, based on the rating of orientation of the characters (digits and capital letters) by French adult students, a clear separation of the left-oriented and right-oriented characters was obtained. The result, in perfect accordance with the rating of the orientation of capitalletter by American students (Treiman et al., 2014), provides an objective basis for discussing many previous results reported by Fischer and coll. (e.g. Fischer \& Tazouti, 2012; Fischer \& Koch, 2016b). The result is important as an obvious limitation of Study 2 and of the theory is their dependency on the culture and educational system. Not only because in some cultures writing runs from right to left, but also because of the order and timing of introduction of manuscript vs. cursive writing (cf. Wolf, Abbott, \& Berninger, 2017), or of uppercase vs. lowercase letters.

The clarity of the separation in left- and right-oriented characters by adult students comes from different sources. The fact that many capital letters ( $B, D, E, F, K, L, N, P$ and $R$ ) consist of a vertical stroke (which can be perceived as a barrier) with a distinctive element to the right of this stroke, is certainly an important cause of their right-orientation appearance (Kolers, 1969; Treiman \& Kessler, 2011). Its counterpart makes appear the digit 1 (and perhaps 7 and 9) left-oriented. The dynamic of writing - from left to right in the western culture of the current 
studies - explains also some other ratings by the adult students. For example, in writing C, $\mathrm{G}$ or $\mathrm{Q}$, the writer is in good position to write the next letter on the right. Fischer (2017) demonstrated that when French children 'spontaneously' write from right to left, they generally reverse the right-facing letters! Because of the static display of the letters in Study 1 , explain the rating of the adult students by the dynamics of writing can appear inappropriate. However, fMRI studies have repeatedly shown that writing motor processes are implicitly evoked even when passively observing letters (Longcamp, Anton, Roth, \& Velay, 2003, 2005).

What about other factors that were assumed explain mirror reversal? In the aim to discuss the role of some individual factors (hand of writing, gender age), a classical multiple regression and many mixed models were computed. The mixed model (M2.2Cod), controlling for by-item variability (two classes of digits: LO and RO), and including, two fixed factors (hand of writing and gender), yielded hand of writing as a significant factor predicting reversal. This seems, a priori, contradictory with the claim by Fischer and Koch (2016a) that left-hand writing is not the explanation of reversal. Even if the present research only includes $n=29$ children who wrote with their left hand, its power, calculated with the harmonic mean of the two sub-samples ( $n^{\prime}=52.36$ : see Cohen, 1988, p. 42), is not far from that of the study by Fischer and Koch $(n=59)$. For example, with a bilateral t-test at the .05 level, the power for detecting a $d=.50$ median difference were .714 and .765 in these two studies, respectively. Thus, the present result should not be ignored. However, it is not contradictory with the claim by Fischer and Koch (2016a). Many reasons can be put forward in support of this non-contradiction.

A major reason is the convergence of the two studies in their finding that the two subsamples, determined by the hand of writing, do not differ in the digits they reversed mostly: in particular, children who wrote with the left hand, as children who wrote with the right hand, reversed the digit 3 most, and the digit 4 least, in both studies. The material was not the same in the two studies: Fischer and Koch studied both capital letters and digits, and mirror writing of the name, whereas the current research was restricted to digit reversal. In Fischer and Koch's study, there was also a slight non-significant greater percentage reversal in the left-hand sub-sample (28.61\%) compared to the right-hand sub-sample (25.94\%). At this regard, it should be remarked that the difference between significant (in the present study) and not significant (in the study, by Fischer and Koch) is not itself necessarily significant (cf. Nieuwenhuis, Forstmann, \& Wagenmakers, 2011, p. 1107).

Because the difference between girls and boys in reversal was only marginally significant, it will not be discussed at length. We would only note that this finding does not support the greater percentage of character reversal by boys than by girls, presented as 'unexpected' by Fischer and Koch (2016a, p. 41).

Finally, a comparison of by-item and by-participant random-intercept mixed models showed that the by-item variance of reversal is considerably greater (about 20 times) than the by-participant variance explained by the hand of writing. When the eight asymmetrical digits are regrouped in two classes - left-oriented vs. right-oriented digits - determined by the ratings of the students in Study 1, the part of by-item random-intercept variance explained by the two classes is greater than when the digits are processed as eight different items, $11.5 \%$ vs. $4.7 \%$ (see Tables 1 and 2, respectively). Thus, the left-orientation of the digits, when combined with a right-writing rule, appears a major explanation of their reversal by typical developing children, even if it does not explain the non-negligible reversals of the digits 4, 5 and 6. 


\section{Acknowledgements}

I would thank all the children, students, teachers, and administrative officials who were involved in the experiments.

\section{Disclosure statement}

No potential conflict of interest was reported by the author.

\section{Funding}

This research did not receive any specific grant from funding agencies in the public, commercial, or not-for-profit sectors.

\section{References}

Baayen, R. H., Davidson, D. J., \& Bates, D. M. (2008). Mixed-effects modeling with crossed random effects for subjects and items. Journal of Memory and Language, 59, 390-412.

Bates, D., Maechler, M., Bolker, B., \& Walker, S. (2015). Fitting linear mixed-effects models using Ime4. Journal of Statistical Software, 67(1), 1-48. doi:10.18637/jss.v067.i01

Borst, G., Ahr, E., Roell, M., \& Houdé, O. (2015). The cost of blocking the mirror generalization process in reading: Evidence for the role of inhibitory control in discriminating letters with lateral mirror-image counterparts. Psychonomic Bulletin \& Review, 22, 228-234.

Burgoon, E. M., Henderson, M. D., \& Markman, A. B. (2013). There are many ways to see the forest for the trees: A tour guide for abstraction. Perspectives on Psychological Science, 8, 501-520.

Cohen, J. (1988). Statistical power analysis for the behavioral sciences (2nd ed.). New York, NY: Taylor \& Francis.

Corballis, M. C., \& Beale, I. L. (1976). The psychology of left and right. New York, NY: Erlbaum.

Dehaene, S. (2010). Reading in the brain: The new science of how we read. London: Penguin Books (first published by Viking Penguin, USA, 2009).

Erlikhman, G., Strother, L., Barzakov, I., \& Caplovitz, G. P. (2017). On the legibility of mirror-reflected and rotated text. Symmetry, 9, 28. doi:10.3390/sym 9030028

Fischer, J. P. (2010). Vers une levée du mystère des écritures en miroir (des chiffres) chez l'enfant [Digit mirror-writing in children: Towards an unlocking of the mystery]. L'Année psychologique, 110, 227-251.

Fischer, J. P. (2011). Mirror writing of digits and (capital) letters in the typically developing child. Cortex, 47, 759-762.

Fischer, J. P. (2013). Digit reversal in children's writing: A simple theory and its empirical validation. Journal of Experimental Child Psychology, 115, 356-370.

Fischer, J. P. (2017). Character reversal in children: The prominent role of writing direction. Reading and Writing, 30, 523-542.

Fischer, J. P., \& Koch, A. M. (2014). La magie computationnelle de la voie ventrale est-elle à l'origine de l'inversion des lettres et des chiffres chez l'enfant de cinq à six ans? [Does the reversal of the letters and digits by the five to six-year-old child originate in the computational magic of the ventral stream?] Revue de Neuropsychologie, 6, 230-237.

Fischer, J. P., \& Koch, A. M. (2016a). Mirror writing in 5- to 6-year-old children:The preferred hand is not the explanation. Laterality: Asymmetries of Body Brain and Cognition, 21, 34-49.

Fischer, J. P., \& Koch, A. M. (2016b). Mirror writing in typically developing children: A first longitudinal study. Cognitive Development, 38, 114-124.

Fischer, J. P., \& Tazouti, Y. (2012). Unraveling the mystery of mirror writing in typically developing children. Journal of Educational Psychology, 104, 193-205.

Fuller, J. K. (1916). The psychology and physiology of mirror-writing. University of California Publications, 
Hildreth, G. (1950). The development and training of hand dominance: IV. Developmental problems associated with handedness. Journal of Genetic Psychology, 76, 39-100.

Kolers, P. A. (1969). Clues to a letter's recognition: Implications for the design of characters. Journal of Typographic Research, 3, 145-168.

Kolers, P. A., \& Perkins, D. N. (1975). Spatial and ordinal components of form perception and literacy. Cognitive Psychology, 7, 228-267.

Kushnir, T., Arzouan, Y., Karni, A., \& Manor, D. (2013). Brain activation associated with practiced left hand mirror writing. Brain and Language, 125, 38-46.

Lewis, E. R., \& Lewis, H. P. (1965). An analysis of errors in the formation of manuscript letters by firstgrade children. American Educational Research Journal, 2, 25-35.

Longcamp, M., Anton, J. L., Roth, M., \& Velay, J. L. (2003). Visual presentation of single letters activates a premotor area involved in writing. Neurolmage, 19, 1492-1500.

Longcamp, M., Anton, J. L., Roth, M., \& Velay, J. L. (2005). Premotor activations in response to visually presented single letters depend on the hand used to write: A study on left-handers. Neuropsychologia, 43, 1801-1809.

McIntosh, R. D., \& Della Sala, S. (2012). Mirror-writing. The Psychologist, 25, 742-746.

MEN. (2013). Modèles d'écriture scolaire [Academic writing styles]. Paris: Ministère de l'éducation nationale (Direction générale de l'enseignement scolaire).

Nieuwenhuis, S., Forstmann, B. U., \& Wagenmakers, E. J. (2011). Erroneous analyses of interactions in neuroscience: A problem of significance. Nature Neuroscience, 14, 1105-1107.

Orton, S. T. (1925). "Word-blindness” in school children. Archives of Neurology and Psychiatry, 14, 581-615.

Pinheiro, J., Bates, D., DebRoy, S., Sarkar, D., \& R Core Team. (2017). n/me: Linear and nonlinear mixed effects models (R package version 3.1-131). Retrieved from https://cran.r-project.org/web/packages/ nlme/nlme.pdf

Puranik, C. S., \& Lonigan, C. J. (2014). Emergent writing in preschoolers: Preliminary evidence for a theoretical framework. Reading Research Quarterly, 49, 453-467.

R Core Team. (2015). R: A language and environment for statistical computing. Vienna: R Foundation for Statistical Computing.

Reed, S. K. (2016). A taxonomic analysis of abstraction. Perspectives on Psychological Science, 11, 817-837.

Rollenhagen, J. E., \& Olson, C. R. (2000). Mirror-image confusion in single neurons of the macaque inferotemporal cortex. Science, 287, 1506-1508.

Schubert, T. M. (2017). Why are digits easier to identify than letters? Neuropsychologia, 95, 136-155.

Shapley, R., Caelli, T., Grossberg, S., Morgan, M., \& Rentschler, I. (1990). Computational theories of visual perception. In L. Spillmann, \& J. S. Werner (Eds.), Visual perception: The neurophysiological foundations (pp. 417-448). San Diego, CA: Academic Press.

Simner, M. L. (1984). The grammar of action and reversals errors in children's printing. Developmental Psychology, 20, 136-142.

Treiman, R., Gordon, S., Boada, R., Peterson, R. L., \& Pennington, B. F. (2014). Statistical learning, letter reversals, and reading. Scientific Studies of Reading, 18, 383-394.

Treiman, R., \& Kessler, B. (2011). Similarities among the shapes of writing and their effects on learning. Written Language and Literacy, 14, 39-57.

Wolf, B., Abbott, R. D., \& Berninger, V. W. (2017). Effective beginning handwriting instruction: Multimodal, consistent format for 2 years, and linked to spelling and composing. Reading and Writing, 30, 299-317. 